\title{
A novel smart grid fault diagnosis algorithm based on optimized BP neural network
}

\author{
Zhang Peng ${ }^{\mathrm{a}, \mathrm{b}, \mathrm{c}}$, Liu Na ${ }^{\mathrm{b}}$, Qu Bo-yang ${ }^{\mathrm{a}}$, , Chang Jing $^{\mathrm{a}}$, Xiao Jun-ming ${ }^{\mathrm{a}}$, Zhao Qi- \\ feng $^{\mathrm{a}}$, Lin Man-man ${ }^{\mathrm{a}}$ \\ ${ }^{a}$ Zhongyuan University of Technology, Zhengzhou, 450007, China. \\ ${ }^{b}$ HaMi Technical College, HaMi 100875, China. \\ ${ }^{c}$ Hami YuXin Energy Industry Research Institute Co., Ltd,Hami 100875, China.
}

\begin{abstract}
Considering that the structure of Smart Grid Fault Diagnosis Algorithm based on BP neural network became complex due to the increase of the sample dimension and the network fell easily into local maximums or minimums, genetic algorithm and rough set were combined to optimize the BP neural network. Rough set was applied to reduce the dimension by attribute significance to simplify the network. Genetic algorithm was introduced to globally search the weights and bios to avoid network falling into the local extremes. Results indicated that prediction accuracy was increased greatly than the traditional BP neural network, and the method is feasible and effective.
\end{abstract}

Keywords: smart grid, Fault Diagnosis, BP neural network, rough set, genetic algorithm, weights and bios.

\section{Introduction}

The study on the smart grid of the Regional Network Centralized Protected Mode(RNCRM) has become one of the important research topic in power system. The study on the Regional Network Centralized Protected Mode has been carried out based on the panoramic data analysis and online dynamic security analysis (DSA)of the smart grid. Recently, the study has attracted wide attention of scholars all over the world. A variety of artificial intelligence technology(AI) should be used to build the smart grid of RNCRM. These AI include expert system (ES), artificial neural network (ANN), fuzzy system theory (FST), genetic algorithm (GA), grey system theory (GS), rough set $[1,2,3]$, and so on. The comprehensive intelligent method should be found based on these AI to realize intelligent fault diagnosis and prediction of smart grid.

Artificial neural networks are often used to solve nonlinear mapping or classification problems in fault diagnosis. And the BP neural network is one of the most mature and widely used ANN. The BP neural network can extract the reasonable rules automatically by learning the training samples, which can have good prediction ability to the test samples $[4,5,6]$.

But, the BP neural network also has some limitations, [3,7,8,9,10]. First, it does not have the preprocessing function for input samples. When the dimension of input sample is large, the structure of neural network is complex too. The training time of the neural network is greatly extended. And the realtime performance of the BP neural network is obviously reduced. Secondly, BP neural network is easy to fall into local optimum. The weights and bios of the BP neural network are modified along the reverse direction of the error function gradient, which belongs to the optimization method of linear search. But, the transfer function is a nonlinear function, and the error function is quadratic function. These functions usually have a lot of local optimums. So, using a linear search method can easily fall into these local

\footnotetext{
* Manuscript received February 4, 2018; revised July 2, 2018.

Corresponding author. E-mail address: zhangpengbbc@163.com doi: $10.12720 /$ sgce.7.3.170-179
} 
optimums, which can also lead to premature convergence, slow convergence, or even stagnation [11,12].

Therefore, this paper proposes a new fault tolerant algorithm for smart grid based on BP neural network. In this paper, rough set and genetic algorithm are applied to BP network to optimize it. Firstly, the rough set is used to reduce the sample space, and then, the reduced samples are then input into the neural network for training. In the training process, the genetic algorithm is used to global search the weights and bios of the network to avoid the local extremum caused by the BP algorithm. The complexity of the BP neural network is reduced, the convergence speed of the network is accelerated, and the prediction accuracy of the neural network is also improved. The new algorithm has been successfully applied to fault diagnosis and intelligent prediction of smart grid.

The remainder of this paper is organized as follows. In the next section, we survey the reduction of sample space by rough sets. The optimization method of weights and bios based on genetic algorithm is introduced in detail. Section IV reports a series of experiments on the application of the new fault tolerant algorithm for smart grid based on BP neural network. The conclusions drawn from this paper are given in Section IX.

\section{Reduction of Sample Space by Rough Sets}

Sample space is called knowledge expression system in rough set. Rough sets mainly study the knowledge that can form division or coverage on the domain $[13,14,15]$. The domain can be interpreted as a set of all sample points in the sample space [2,16,17]. The concept of division and equivalence relation is consistent, that is, the classification of all samples in the sample space is carried out based on equivalence relation. $U / R$ is the set of all equivalence classes of $R$ partition under the equivalence relation $U$. In general, the $R$ equivalence class of element $x$ in domain $U$ is represented by $[x]_{R}$. Given a set of $X$, if $X$ can be expressed as the union of certain equivalence classes of equivalence relation $R, X$ can be called a set of definable sets or exact set of $R$. Otherwise, the set $X$ is called $R$ non-definable set or $R$ rough set. And the rough sets are usually described as upper and lower approximation sets.

$$
\begin{gathered}
\underline{R}(X)=\left\{x \mid(\forall x \in U) \wedge[x]_{R} \subseteq X\right\} \\
\bar{R}(X)=\left\{x \mid(\forall x \in U) \wedge[x]_{R} \cap X \neq \varnothing\right\}
\end{gathered}
$$

where, $\operatorname{pos}_{R}(X)=\underline{R}(X)$ is the $R$ positive domain of $X, n e g_{R}(X)=U-\bar{R}(X)$ is the $R$ boundary domain of $X, n e g_{R}(X)=U-\bar{R}(X)$ is the $R$ negative domain of $X$.

When the attribute set of the sample space (represented by $A$ ) is composed of the conditional attribute (represented by $C$ ) and the decision attribute (represented by $D$ ), the knowledge expression system is the decision table. In general, there is a certain degree of dependence between the conditional attributes and the decision attributes, which is defined by dependence:

$$
\gamma_{C}(D)=\frac{\left|\operatorname{pos}_{C}(D)\right|}{|U|}=\frac{|\underset{X \in U / D}{\cup} \underline{C}(X)|}{|U|}
$$

where, $\operatorname{pos}_{C}(D)$ is the $C$ positive domain of $D, g_{C}(D)$ measures the knowledge based on the attribute $C$. The objects in the domain can be correctly divided into decision classes.

When the attribute reduction of decision table is usually used, the concept of attribute importance degree is often used. In the attribute reduction of decision table, the concept of attribute importance is often used. It is defined as: $\operatorname{sig}(a, B ; D)=g_{\{a \cup B\}}(D)-g_{\{B\}}(D)$, which represents the importance of the attribute $a$ to the attribute $B$ relative to the decision attribute $D$.

Because most of the conditional attributes have dependency and relevance, not all conditional attributes are necessary for attribute decision making, which leads to the attribute reduction problem. In the premise 
of ensuring the classification ability unchanged, it can simply represent the dependence and association of decision attributes in the decision table to the conditional attribute set.

\subsection{Solving process of relative reduction of decision table.}

Generally, the relative reduction of decision tables contains kernel attributes, and the intersection of relative reductions is the kernel function $[1,17,18]$. Therefore, in the decision table relative reduction algorithm, the relative kernel of the decision table should be obtained first. For " $R$ ? $C$, if $R$ satisfies function, $\operatorname{pos}_{\{C-R\}}(D)=\operatorname{pos}_{C}(D), R$ is called $D$ unnecessary in $C$, otherwise $R$ is necessary for $C$ in $D$. The set of all primitive relations necessary for $D$ in $C$ is called the kernel of $D$ relative to $C$, denoted as $\mathrm{CORE}_{D}(C)$.

The relative kernel of some decision tables is the relative reduction. When the relative kernel is found, it should be judged whether it is a reduction or not. For the nonempty set $P$ of the conditional attribute $C$, if the condition is satisfied: $(1) \operatorname{pos}_{P}(D)=\operatorname{pos}_{C}(D)$.(2)For any $R$ Í $P$, there is a formula $\operatorname{pos}_{R}(D)^{1} \operatorname{pos}_{C}(D)$. Then $P$ is called a reduction of $D$ on $C$, which can be denoted as $R E D_{D}(C)$.

If reduct $=\operatorname{CORE}_{D}(C)$, then the redundant attribute set can be represented as redundant $=C-C O R E_{D}(C)$. If $\operatorname{pos}_{\text {reduct }}(D)=\operatorname{pos}_{C}(D)$ is established, the set reduct (at this time, it is the relative kernel) is a relative reduction of the decision table. Otherwise, each attribute of the redundant attribute set should be added to the set reduct sequentially. According to the definition of attribute importance, we can find the corresponding attribute $\alpha_{i}$ which reaches the maximum value. If reduct $=$ reduct $\bigcup\left\{\alpha_{i}\right\}$ is established, $\mathrm{B}$ redundant $=$ redundant $-\left\{\alpha_{i}\right\}$ is used to update the set of reduct to be measured and redundant attribute set redundant. Continue the above operation until the $\operatorname{pos}_{\text {reduct }}(D)=\operatorname{pos}_{C}(D)$ is established, the obtained set reduct is a relative reduction of the decision table.

\subsection{Optimization of BP neural network structure based on Rough Sets.}

The rough set is introduced into the fusion model of BP neural network as its front-end system. The redundant attributes can be eliminated. The relative reduction attribute set of sample space can be obtained. The dimension of sample space can be reduced. The structure of the neural network can be simplified too. In addition, the neural network can filter the random noise of the parameter in the sampling process to a certain extent. The rough set is sensitive to noise. The combination of the neural network and rough set can not only improve the real-time performance of the system, but also help to enhance the fault tolerance of the system. The specific steps of improving BP neural network based on rough set are as follows:

(1) Discretization and normalization of the original sample space to obtain the original decision.

(2) Reduce the decision table, eliminate the redundant attributes, obtain the reduction decision table by using the attribute importance reduction algorithm.

(3) The training samples of the decision table are input into the BP neural network to train again, and the weights and bios are updated successively until the given accuracy is satisfied.

(4) Using the test samples in the sample space to test the trained BP neural network, and get the prediction results.

\section{Optimizing the Weights and Bios Based on Genetic Algorithm}

The optimized weights and bios of the BP neural network are encoded as individuals of the genetic algorithm. The sum of the absolute values of the BP network prediction error is used as the fitness value of the genetic algorithm. The optimal individual is selected by iterative operation of genetic algorithm $[11,19,20]$. The optimal weight and threshold of the network can be obtained by decoding the optimal individual $[21,22,23]$. Specific algorithm practice is as follows:

(1) The parameters of genetic algorithms should be initialized, such as the number of iterations, 
population size, crossover probability, mutation probability, the length and range of individuals in the population, fitness function, etc. The weights and bios of each layer in the BP network are encoded as individuals of the genetic algorithm, and the fitness values of all individuals should be calculated.

(2) The selection probability and cumulative probability are calculated according to the fitness value of each individual. The excellent individuals are selected from the parent population to form a new species group based on the roulette wheel algorithm.

(3) Two individuals should be selected from the new species group, and new individuals are obtained by cross operation according to a certain probability.

(4) An individual was selected from the new species group, and then, A new individual is obtained by mutation operation according to a certain probability.

(5) The fitness value of the latest population is calculated to find the optimal and worst individual. Then the worst individual is replaced with the best individual to preserve the optimal individual and its fitness value.

(6) When the number of iterations arrives, stop the optimization operation, otherwise jump to (3) step and continue to optimize calculation.

\subsection{Coding of genetic individuals.}

There are two kinds of individual encoding methods in genetic algorithm: binary encoding and real coding[24,25]. In the optimization calculation, binary encoding methods requires discretization of the continuous space, which will cause a certain encodeing error. Real coding method does not exist discrete error, and has the advantages of intuitive and high precision. Therefore, this paper adopts the real coding method.

BP neural network is composed of input layer, hidden layer and output layer. Therefore, the individual consists of four parts: the weights and bios from the input layer to the hidden layer, the weights and bios form the hidden layer to the output layer. In this paper, the the weights and bios of the hidden layer are denoted as $w_{1}$ and $b_{1}$, and the the weights and bios of the output layer are denoted as $w_{2}$ and $b_{2}$. Where, $w_{1}, b_{1}, w_{2}, b_{2}$ are all matrix or vector, and the corresponding elements are all real numbers. The specific dimension of the $w_{1}, b_{1}, w_{2}, b_{2}$ is determined by BP neural network structure.

\subsection{Construction method of fitness function.}

Training samples are input into BP neural network to predict the system output $[26,27,28]$. The sum of the absolute value of the error between the actual output and the expected output is used as the fitness value of the individual.

$$
F=k\left(\sum_{i=1}^{n} a b s\left(y_{i}-o_{i}\right)\right)
$$

Where, $n$ is the output connection points for BP neural network, $y_{i}$ is the expected output of the $i$ node of the BP neural network, $o_{i}$ is the predicted output of the $i$ node, and $k$ is the coefficient of calculation. The smaller the fitness value of the BP neural network is, the better the network prediction ability is. Note: in actual iterations, the reciprocal is usually taken as the fitness value.

\subsection{Selection operation.}

In this paper, the roulette method is used to solve the selection operation. Firstly, the selection probability of each individual is calculated according to the fitness value of all the individuals in the parent population. Then, according to the iteration of the population size, each solution cycle generates a random number of [ $\left[\begin{array}{ll}0 & 1\end{array}\right]$. Then, the cumulative probability is calculated. When the cumulative probability is greater than or equal to the random number, the corresponding individuals are selected. The calculation is repeated until the iteration is stoped. And the formula of selection probability is as follows: 


$$
p_{i}=\frac{\left(k / F_{i}\right)}{\sum_{i}^{N}\left(k / F_{i}\right)}
$$

Cumulative probability is:

cumul $_{i}=$ cumul $_{i-1}+p_{i}$

Where, $F_{i}$ is the fitness value of individual $i$, The fitness value is reciprocal before the general individual selection operation. And $k$ is the coefficient of calculation, $N$ is the number of individuals in the species group.

\subsection{Crossover operation}

The crossover probability $P_{c}$ in crossover operation has a direct impact on the convergence and effectiveness of genetic algorithm. The greater the probability is, the faster a new individual is generated, but the probability of genetic algorithm degradation into random search is also greater. The smaller the probability, the slower the search speed, and even stagnation. Therefore, the adaptive crossover probability is used in this paper, as shown in the following formula.

$$
P_{c}=\left\{\begin{array}{c}
P_{c 1}-\frac{\left(P_{c 1}-P_{c 2}\right)\left(f^{\prime}-f_{\text {avg }}\right)}{f_{\max }-f_{\text {avg }}}, \quad f^{\prime} \geq f_{\text {avg }} \\
P_{c 1}, \quad f^{\prime}<f_{\text {avg }}
\end{array}\right.
$$

Where, $f_{\text {avg }}$ is the average fitness value of each generation population, $f_{\max }$ is the maximum fitness value of each generation population, and $f^{\prime}$ is the larger fitness value of the two individuals to be crossed. When the $f^{\prime}$ 'is greater than or equal to $f_{\text {avg }}$, the cross probability is reduced, Otherwise, cross probability is increased.

When the crossover probability is satisfied, the crossover operations of the $k$ chromosome $a_{k}$ and the $l$ chromosome $a_{l}$ at the $j$ locus are as follows:

$$
\left\{\begin{array}{l}
a_{k j}=a_{k j}(1-b)+a_{l j} b \\
a_{l j}=a_{l j}(1-b)+a_{k j} b
\end{array}\right.
$$

Where, $b$ is a random number.

\subsection{Mutation operation}

Like the crossover probability, the probability of mutation $\left(P_{m}\right)$ also has a direct impact on the convergence and effectiveness of genetic algorithm. If $P_{m}$ is too large, the genetic algorithm will degenerate into a random search algorithm. If $P_{m}$ is too small, it is difficult to produce new individual genes. Therefore, the adaptive mutation probability is used in this paper, as shown in the following formula.

$$
P_{m}=\left\{\begin{array}{c}
P_{m 1}-\frac{\left(P_{m 1}-P_{m 2}\right)\left(f^{\prime}-f_{\text {avg }}\right)}{f_{\max }-f_{\text {avg }}}, \quad f^{\prime} \geq f_{\text {avg }} \\
P_{m 1}, \quad f^{\prime}<f_{\text {avg }}
\end{array}\right.
$$

Where, the meaning of $f_{\text {avg }} 、 f_{\max } 、 f^{\prime}$ is the same as that in the preceding part of the text.

When the mutation probability is satisfied, the mutation location of the individual is selected randomly.For example, the $j$ chromosome $a_{i j}$ of the $i$ individuals was selected for mutation, and the mutation operation was as follows.

$$
a_{i j}= \begin{cases}a_{i j}+\left(a_{i j}-a_{\max }\right) * f(g) & r>0.5 \\ a_{i j}+\left(a_{\min }-a_{i j}\right) * f(g) & r \leq 0.5\end{cases}
$$


Where, $a_{\max }$ is the upper limit of the range of chromosome $a_{i j}, a_{\min }$ is the lower limit of chromosome $a_{i j}, f(g)=r_{2}\left(1-g / G_{\max }\right)^{2}, r_{2}$ is a random number, $g$ is the current number of iterations, $G_{\max }$ is the total number of iterations, and $r$ is a random number between 0 and 1 .

Optimal operation of genetic algorithm will produce the optimal individual. That is, the optimal weights and bios are used as the structural parameters of BP neural networks. Furthermore, the test sample is used to verify the prediction ability and accuracy of the BP neural network [29, 30, 31].

\section{Simulation and Experiments}

The accident decision record data set is used as the decision table in the auxiliary decision making system of an online dispatching assistant decision system for a regional power grid. First, rough sets are used to solve the reduction decision table. Then, the reduction decision table is input into BP neural network for training. The genetic algorithm is used to search the weights and bios of the network in the training process. The rough set can only deal with discrete data, and the accident decision record data set contains discrete and continuous type data. In this paper, in order to obtain the original decision table, we use the algorithms in $[8,11,12]$ to discretize the accident decision record data.As shown in Table 1, there are 11 columns of conditional attributes in the data set, and there are only one of decision result attributes. The samples can be divided into 3 categories. There are 150 samples in all. There are 50 samples of the first class, 53 samples of second types and 47 samples of third classes.

Table1. Fault data decision table after discretization

\begin{tabular}{cccccccccccc}
\hline 1 & 2 & 3 & 4 & 5 & 6 & 7 & 8 & 9 & 10 & 11 & $\mathrm{y}$ \\
\hline 2 & 1 & 2 & 1 & 7 & 2 & 2 & 1 & 2 & 2 & 2 & 1 \\
2 & 1 & 1 & 1 & 7 & 2 & 2 & 1 & 1 & 2 & 2 & 1 \\
$\ldots$ & $\ldots$ & $\ldots$ & $\ldots$ & $\ldots$ & $\ldots$ & $\ldots$ & $\ldots$ & $\ldots$ & $\ldots$ & $\ldots$ & $\ldots$ \\
2 & 1 & 2 & 1 & 8 & 2 & 2 & 1 & 2 & 2 & 2 & 1 \\
1 & 1 & 1 & 1 & 1 & 1 & 1 & 1 & 1 & 1 & 2 & 2 \\
1 & 1 & 1 & 1 & 8 & 1 & 1 & 2 & 1 & 1 & 2 & 2 \\
$\ldots$ & $\ldots$ & $\ldots$ & $\ldots$ & $\ldots$ & $\ldots$ & $\ldots$ & $\ldots$ & $\ldots$ & $\ldots$ & $\ldots$ & $\ldots$ \\
1 & 2 & 1 & 2 & 1 & 1 & 2 & 2 & 1 & 1 & 1 & 2 \\
1 & 1 & 1 & 1 & 9 & 1 & 1 & 1 & 1 & 2 & 1 & 3 \\
1 & 2 & 2 & 2 & 8 & 1 & 1 & 1 & 1 & 2 & 1 & 3 \\
$\ldots$ & $\ldots$ & $\ldots$ & $\ldots$ & $\ldots$ & $\ldots$ & $\ldots$ & $\ldots$ & $\ldots$ & $\ldots$ & $\ldots$ & $\ldots$ \\
2 & 2 & 2 & 2 & 3 & 1 & 1 & 2 & 1 & 2 & 1 & 3 \\
\hline
\end{tabular}

\subsection{Seeking the relative reduction of decision table.}

Attribute reduction for decision table is carried out based on attribute importance algorithm.

(1) Solving the relative kernel of the original decision table, $B$ :

$B=\operatorname{CORE}_{c}(D)=\{7,13\}=\{$ Flavanoids, Proline $\}$, Redundant attribute set $R=\{1,2,3,4,5,6,8,9,10,11\}$. $\operatorname{pos}_{C}(D)=\{u 1\} \bigcup\{u 2\} \bigcup \cdots \cup\{u 150\}=U$, and $\operatorname{pos}_{B}(D)=\varnothing$,

(2) Estimating whether relative kernel $B$ is the relative reduction of original decision table.

$\operatorname{pos}_{C}(D)=\{u 1\} \bigcup\{u 2\} \bigcup \cdots \bigcup\{u 150\}=U, \operatorname{pos}_{B}(D)=\varnothing, \operatorname{pos}_{B}(D)^{1} \quad \operatorname{pos}_{C}(D)$. So, the relative nuclear $B$ is not a relative reduction of the decision table.

(3) If the relative kernel is not the relative reduction of the decision table, the new attribute set is obtained by adding the largest attribute according to the attribute importance of the relative attributes of the redundant attributes. And then the relative reduction can be judged. In this paper, the relative reduction of decision table is reduct $=\{1,5,7,10,11\}$.

A new reduction decision table is constructed by using the attribute column and decision attribute list 
corresponding to the relative reduction reduct. And the new reduction decision table taken as the sample space of the BP neural network.

\subsection{Constructing BP neural network.}

In this paper, a three-layer BP neural network is constructed. The attribute $\{1,5,7,10,11\}$ of the discrete decision table is used as input of the BP network. And the decision attribute column is used as the output of BP neural network. As the total number of samples was 150, 90 samples were randomly selected as training samples, and the remaining 60 samples were used as test samples. The training samples were input into the BP neural network for training according to the 90-row data corresponding to the $\{1,5,7,10,11\}$. So, the input vector of the network is reduced from the original 11 dimensions to the 5 dimensions. The decision attribute values are 1,2,3, which represent three categories of fault record data sets. Therefore, the output layer of the BP neural network uses "Pureline" linear function, the number of nodes is 1 . The hidden layer uses the transfer function as "sigmoid". The number of neurons in the hidden layer is between 3 12 by using the nodes number empirical formula of input and output layer. First, a BP neural network with variable number of nodes can be designed, Then, through error comparison, it is found that when the number of nodes in hidden layer is 10 , the error is minimum. Therefore, the final BP neural network structure contains 5 nodes in the input layer, 10 nodes in the hidden layer, and 1 nodes in the output layer

\subsection{Searching weights and bios of networks based on genetic algorithm.}

\section{(1) Real number coding of individual}

The structure of BP neural network is 5-10-1. So, the weight matrix $\left(w_{1}\right)$ of the input layer and the hidden layer is the matrix of $10 * 5$, and the threshold vector $b_{1}$ is a10* 1 vector. The weight matrix $w_{2}$ of the hidden layer and the output layer is a $10^{*} 1$ vector. The threshold vector $b_{2}$ is a real number. Therefore, the individual of the genetic algorithm consists of 10? 5 10? 1 10? 1 1=71 real numbers. The value range of each real number is defined as [-3 3].

(2) Initialization of parameters of genetic algorithm.

The number of iterations was 100 , and the total number of individuals in the population was 10 . The cross-probability value $P_{c 1}$ is 0.9 . The value of $P_{c 2}$ is 0.6 . The variation probability value $P_{m 1}$ is 0.1 . The value of $P_{m 1}$ is 0.001 .

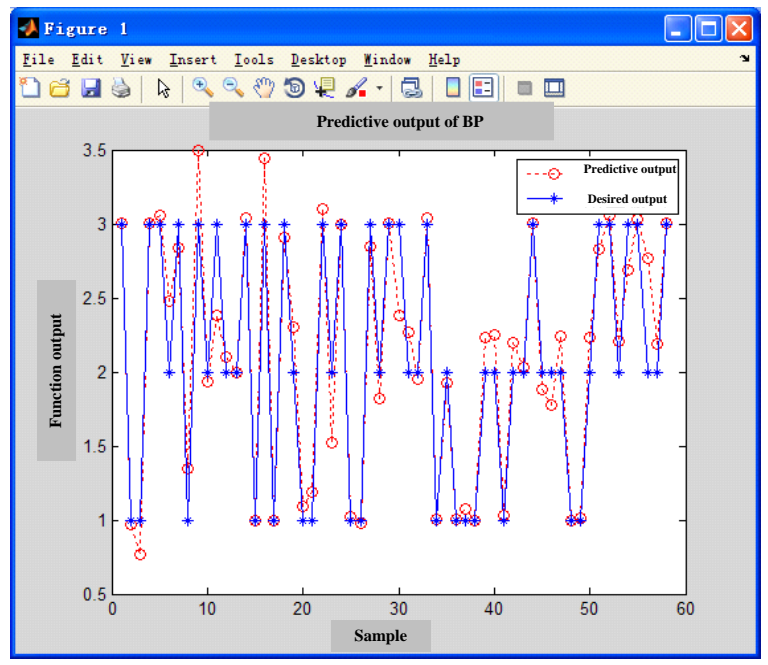

(a) 


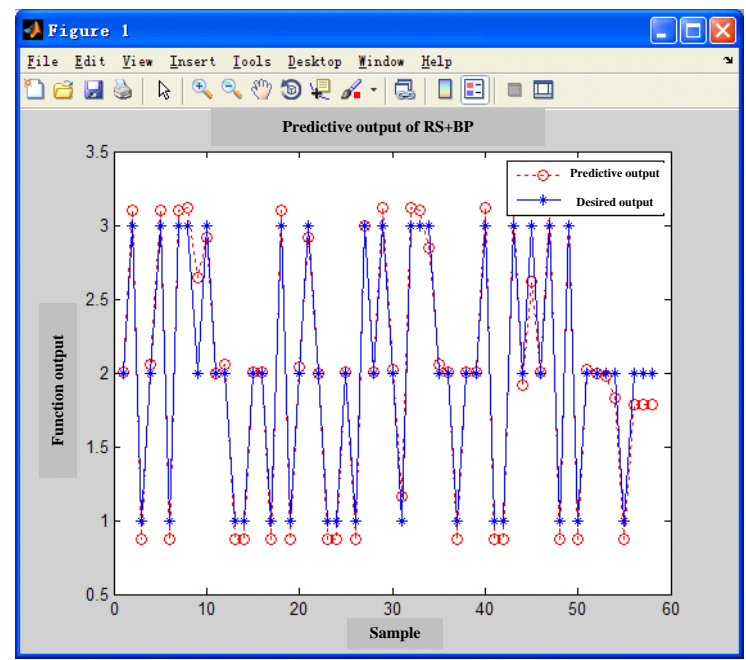

(b)

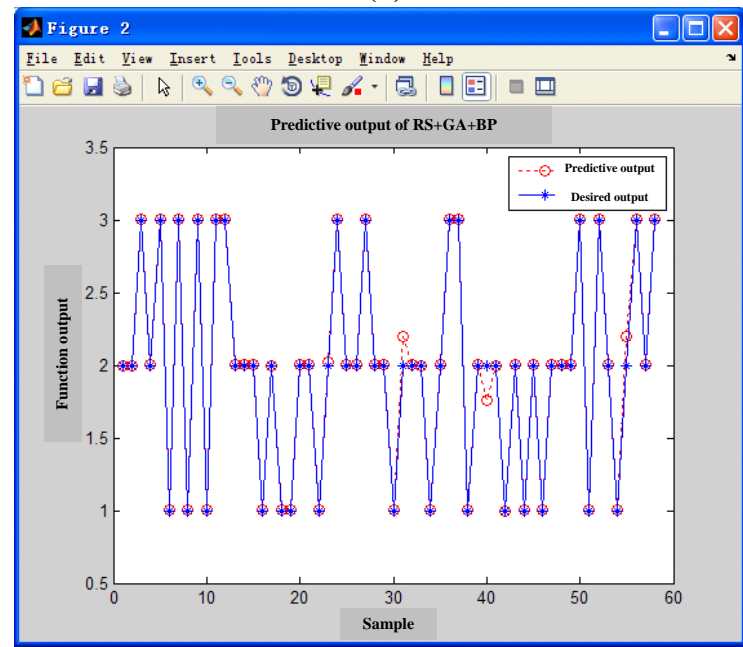

(c)

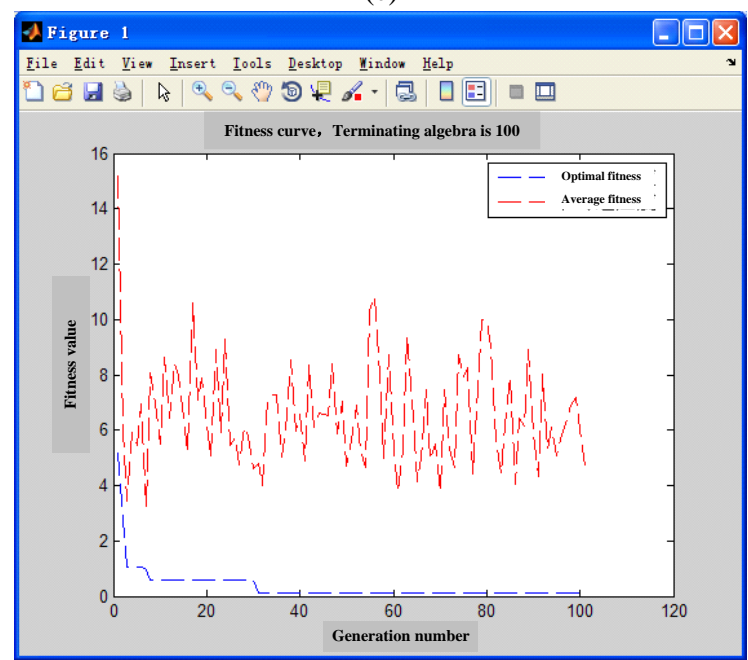

(d)

Fig.1 Prediction output and adaptive curve from three different methods of genetic algorithms. 
The selection, crossover, mutation and fitness value can be calculated according to the formula of the upper section. In the iterative process of genetic algorithm, the four steps are continuously executed to screen out the optimal individuals and the optimal fitness values. And it is used to replace the worst individual and the worst fitness until the iteration step satisfies the set point. The final optimal individual value is the optimal value of each layer weight and threshold value of BP neural network.

\subsection{Analysis of prediction results}

The original decision table is trained by using BP neural network, rough set and BP network integration, and optimizing BP Neural Network Based on Genetic Algorithm and Rough Set. In the same way, test samples are used to predict, and the prediction results are shown in Fig. 1 and Table 2.

The predicted outputs using the BP neural network, RS+BP neural network and RS+GA+BP neural network are displayed in Fig. 1(a), Fig. 1(b), Fig. 1(c). It is obvious that the predicted output in Fig. 1(c) matches the expected output best. The predicted output in figure 1(b) matches the expected output a little better than that in Fig. 1(a). The sample space contains too many redundant attributes, so the prediction result in Fig. 1(a) is not ideal.

Table 2. Prediction error and time consuming from three different methods.

\begin{tabular}{lll}
\hline Algorithm & Mean square error & Consumption time $(\mathrm{s})$ \\
\hline BP & 1.72208 & 0.64048 \\
RS+BP & 1.03605 & 0.46594 \\
\hline RS+GA+BP & 0.23509 & 97.8072
\end{tabular}

From Table 2, it can be found that the prediction error in Fig. 1(b) is smaller than that in Fig. 1 (a), the time consumption in Fig. 1(b) is also shorter than that in Fig. 1(a). The prediction error in Fig. 1(c) is the smaller than the former. But the computation process takes a little longer time. This is mainly caused by the iteration of genetic algorithm.

\section{Conclusion}

A new Smart Grid Fault Diagnosis Algorithm of Optimizing BP Neural Network Based on Genetic Algorithm and Rough Set is proposed in this paper. The rough set is used to reduce the decision table, which can reduce the dimensionality of fault sample space. It not only retains the recognition effect of BP neural network alone, but also simplifies the network complexity and accelerates the convergence speed of the network. Genetic algorithm is introduced to give full play to the characteristics of global optimization of genetic algorithm. The weights and bios are searched in the global range to avoid the BP neural network falling into local extreme points, resulting in network training failure. Experiments show that the optimization of BP neural network based on rough set and genetic algorithm improves the prediction accuracy of BP neural network, enhances the generalization ability of the network, and has higher accuracy. The model shows that the algorithm has a wide application prospect in fault diagnosis and prediction of power system.

\section{Acknowledgement}

This research is supported by the Department of Education Fund Project of Henan Province, China (Project nos.12B510037 and 13B510296), by the Department of Science \&Technology Fund Project of Henan Province, China (Project nos. 162102210092 and 142102210579), and by the Zhengzhou Administration of Science \& Technology Fund Project of Henan Province, China (Project no. 141PPTGG363), and by the Hami Administration of Science \& Technology Fund Project of XinJiang Province, China.

\section{References}

[1] Qiwei H, Salem C, Sajid S, Ashraf L. Spare parts classification in industrial manufacturing using the dominance-based rough set approach. European Journal of Operational Research, 2017, 262(3):1136-1163. 
[2] Jingjing S, Eric CCT, Degang C, Xibei Y. Minimal decision cost reduct in fuzzy decision-theoretic rough set model. Knowledge Based Systems, 2017, 126:104-112.

[3] Rui-sheng L, Yan-bin L, Feng-quan Z. The functional frame and design Principles of smart substation. Power System Protection and Control, 2010; 38(21):24-27.

[4] Ricardo EM, Juan LC. The definition of a measure of artificial neural network design quality based on a Statistical Index of Quality. Neurocomputing, 2017; 255: 71-76.

[5] Abdullah C, Mehmet EY, Hasan B, Alper B. Performance improvement of deep neural network classifiers by a simple training strategy. Engineering Applications of Artificial Intelligence, 2018;67: 14-23.

[6] Davood Z, Reza M, Hadi SY. Sparse Bayesian similarity learning based on posterior distribution of data. Engineering Applications of Artificial Intelligenc, 2018, 67: 173-186.

[7] Cárdenas-Peña D, Collazos-Huertas D, Álvarez-Meza A, Castellanos-Dominguez G. Supervised kernel approach for automated learning using General Stochastic Networks. Engineering Applications of Artificial Intelligence, 2018;68:10-17.

[8] Gang B, Zhigang Z. Region stability analysis for switched discrete-time recurrent neural network with multiple equilibria. Neurocomputing, 2017; 249:182-190.

[9] Mutsumi K, Ryohei M, Sumio S, Tokiyoshi M, Tomoya K, Yasuhiko N. Cellular neural network formed by simplified processing elements composed of thin-film transistors. Neurocomputing, 2017; 248: 112-119.

[10] Seulki L, Seoung BK. Time-adaptive support vector data description for nonstationary process monitoring. Engineering Applications of Artificial Intelligence, 2018;68:18-31.

[11] Qian Z, Hao-na W, Zhi-hong F, Xing-zhe H, Yong W. An approach with filters-neural network for harmonic detection. Power System Protection and Control, 2015; 9(12):42-47.

[12] Yaguo L, Naipeng L, Liang G, Ningbo L, Tao Y, Jing L. Machinery health prognostics: A systematic review from data acquisition to RUL prediction. Mechanical Systems and Signal Processing,2018;104: 799-834.

[13] Congcong C, Feng J, Chifu Y, Seungmin R, Weizheng S, Shaohui L, Zhiguo L. Hyperspectral classification based on spectral-spatial convolutional neural networks. Engineering Applications of Artificial Intelligence, 2018;68: 165-171.

[14] Wei H, Yongguang Y, Wenjuan G. Parameter estimation of fractional-order arbitrary dimensional hyperchaotic systems via a hybrid adaptive artificial bee colony algorithm with simulated annealing algorithm. Engineering Applications of Artificial Intelligence, 2018; 68: 172-191.

[15] Sinh DM, Long TN. Multiple kernel approach to semi-supervised fuzzy clustering algorithm for land-cover classification. Engineering Applications of Artificial Intelligence, 2018;68: 205-213.

[16] Peng Z, Jing C, Boyang Q, and Qifeng Z. Denoising and Trend Terms Elimination Algorithm of Accelerometer signals, Mathematical Problems in Engineering; 2016:1-9.

[17] Haidar S. Evaluation of digital metering methods used in protection and reactive power compensation of micro-grids. Renewable and Sustainable Energy Reviews,2016; 62:260-279.

[18] Jinyue Y, Yongping Z, Priyantha W, Abdul MM, Pietro EC. Renewable energy integration with mini/micro-grids. Applied Energy, 2017; 201:241-244.

[19] Rashad MK. Hybrid and coordinated soft starting controller for wind generation system runs in the standalone Micro Grid. Sustainable Energy, Grids and Networks, 2016; 5:105-113.

[20] Saulo Henrique da Mata, Paulo Roberto Guardieiro. Resource allocation for the LTE uplink based on Genetic Algorithms in mixed traffic environments. Computer Communications, 2017; 107:125-137.

[21] Prasanna D, Vadana, Sasi KK. Autonomous control of smart micro grid in islanding mode. Procedia Technology,2015; 21: 204-211.

[22] Aghajani GR, Shayanfar HA, Shayeghi H. Presenting a multi-objective generation scheduling model for pricing demand response rate in micro-grid energy management. Energy Conversion and Management, 2015; 106:308-321.

[23] Datta A, Ray A., Mukherjee D, Saha H. Selection of islanding detection methods based on multi-criteria decision analysis for grid-connected photovoltaic system applications. Sustainable Energy Technologies and Assessments, 2014; 7:111-122.

[24] Jawahar N, Subhaa R. An adjustable grouping genetic algorithm for the design of cellular manufacturing system integrating structural and operational parameters. Journal of Manufacturing Systems, 2017; 44(1):115-142.

[25] Xuan Z, Xiao-qing H, Wen-ping Q, Peng W. Past, today and future development of micro-grids in China. Renewable and Sustainable Energy Reviews, 2015; 42: 1453-1463.

[26] Abbas R, Eric P, Valeriu V, Rabih A, Hassan F. Selection of discriminant mid-infrared wavenumbers by combining a naïve Bayesian classifier and a genetic algorithm: Application to the evaluation of lignocellulosic biomass biodegradation, Mathematical Biosciences, 2017; 289:153-161.

[27] Sohrab M, Dalila MS, Mohd. WM, Mohd. HH, Kimia G. Modeling and simulation of a communication-assisted digital protection scheme for micro-grids. Renewable and Sustainable Energy Reviews, 2016; 57: 867-878.

[28] Hamed B, Youmin Z, Henry H. Fault-tolerant cooperative control in an offshore wind farm using model-free and model-based fault detection and diagnosis approaches. Applied Energy, 2017; 201:284-307.

[29] Xiangning L, Rui Z, Ning T, Xianshan L, Ming L, Dexian Y. Regional protection scheme designed for low-voltage microgrids. International Journal of Electrical Power \& Energy Systems, 2015; 64: 526-535.

[30] Andrew WKT, Gavin P, Dikai L. A comprehensive approach to real-time fault diagnosis during automatic grit-blasting operation by autonomous industrial robots. Robotics and Computer-Integrated Manufacturing, 2018; 49: 13-23.

[31] Leo R, Sibi S, Milton RS. Distributed optimization of solar micro-grid using multi agent reinforcement learning. Procedia Computer Science, 2015; 46: 231-239. 